\title{
Editorial der GastherausgeberInnen
}

Lange Zeit war das in den 1930er-Jahren entwickelte Bruttoinlandsprodukt (BIP) der dominante Maßstab für das Entwicklungsniveau einer Gesellschaft. Doch spätestens seit Ende der 197oer-Jahre mehrten sich kritische Stimmen: Kann eine einzige ökonomische Kennziffer ein hinreichender Indikator für die gesamtgesellschaftliche Entwicklung und den Fortschritt im Allgemeinen sein? Werden dabei nicht zentrale Aspekte und Vorbedingungen ausgeblendet? Die entsprechenden Vorbehalte wurden unter anderem damit begründet, dass das BIP keine Aussagen zur tatsächlichen Verteilung des Wohlstands innerhalb einer Gesellschaft zulasse und somit gegebenenfalls über signifikante Ungleichheiten hinwegtäusche. In jüngerer Vergangenheit wurde darüber hinaus angemerkt, dass auf der Grundlage des BIP keine Einschätzungen über die Nachhaltigkeit einer Volkswirtschaft möglich seien, was in den einschlägigen Debatten den Ruf nach besseren Alternativen lauter werden ließ.

Im entwicklungspolitischen Kontext wurde zu Beginn der 1990er-Jahre versucht, dieser Kritik mit der Einführung des mehrdimensionalen Human Development Index (HDI) in die Berichterstattung des Entwicklungsprogramms der Vereinten Nationen zu begegnen. Die gemeinsame Berücksichtigung des kaufkraftbereinigten Bruttonationaleinkommens pro Kopf, der durchschnittlichen Lebenserwartung bei Geburt sowie des Bildungsniveaus sollte das tatsächliche Wohlergehen der jeweiligen Bevölkerung stärker in den Mittelpunkt internationaler Vergleiche rücken.

In Europa wurde unter anderem im Rahmen der Initiative „Beyond GDP“ das Ziel formuliert, neue Indikatoren zu entwickeln, „die so [...] ansprechend wie das BIP sind, aber die umweltbezogenen und sozialen Aspekte des Fortschritts in höherem Maße miteinschließen“ (Europäische Kommission 2015). Beinahe zeitgleich wurde durch den damaligen französischen Präsidenten Sarkozy die Commission on the Measurement of Economic Performance and Social Progress ins Leben gerufen, der mit Joseph Stiglitz und Amartya Sen unter anderem zwei Wirtschaftsnobelpreisträger angehörten. Auch ihr ging es darum, die Grenzen des BIP als Indikator der wirtschaftlichen Leistungsfähigkeit sowie des sozialen Fortschritts in den Blick zu nehmen, um daran anschließend der Frage nachzugehen, wie bessere Indikatoren aussehen könnten. Die OECD griff einige der daraus resultierenden Überlegungen in ihrer „Better Life“-Initiative auf. Diese hat mittlerweile in Form der „Wie geht's Österreich?“Berichte auch eine Entsprechung und Vertiefung auf nationaler Ebene erfahren. Und auch der kürzlich im siebten Jugendbericht veröffentlichte „Better-Life-Index Jugend“ knüpft an die Überlegungen der OECD an.

Well-Being ist im Zusammenhang mit den oben genannten Aktivitäten zu einer zentralen Bezugsgröße avanciert, über deren Relevanz grundsätzlich breiter Konsens besteht. Im Gegensatz zum BIP ist es bislang jedoch noch nicht gelungen, das Konzept allgemeingültig zu operationalisieren. Vor diesem Hintergrund erscheint bereits die Übersetzung des Begriffs ins Deutsche problematisch. Soll sie unter Betonung seines objektiven Charakters mit „Wohlergehen“, stärker subjektiv gefärbt mit „Wohlbefinden“ oder einfach mit „Wohl“ bzw. „Wohlfahrt“ erfolgen, oder soll der Begriff überhaupt unübersetzt bleiben?

Darüber hinaus richtete die Suche nach Alternativen zum BIP in diversen Fachrichtungen die Aufmerksamkeit auf große, bislang kaum in den Blick genommene Wissensbestände. Je nachdem aus welchem disziplinären Blickwinkel Well-Being beleuchtet wird, lässt sich augenscheinlich eine beliebig große Anzahl an „Bindestrich“Begriffen generieren, wie etwa „social“ oder „psychological well-being“. Zudem steht das Konzept in engem Zusammenhang mit Begriffen wie Gesundheit, Wohlstand (bzw. Armut), Zufriedenheit und Glück, was oft zu unscharfen Abgrenzungen führt. Einigkeit scheint es lediglich hinsichtlich einiger formaler Aspekte zu geben, wie beispielsweise dass es jedenfalls mehrdimensional zu messen sei und dabei grob zwischen subjektiven (z. B. durch

2017 | innsbruck university press Innsbruck

Momentum Quarterly I ISSN 2226-5538 I momentum-quarterly.org

Vol. 6, No 2 I DOI 10.15203/momentumquarterly.vol6.no2.p63-64 
Zufriedenheitsbefragungen gewonnenen) und objektiven (z. B. in Form von amtlichen Statistiken vorliegenden) Maßen unterschieden werden könne; wobei allerdings schon wieder Uneinigkeit darüber herrscht, ob und wie diese Maße zueinander in Beziehung gesetzt werden sollten. Trotz - oder vielleicht auch gerade wegen - dieser Unschärfen erfährt das Konzept nicht zuletzt in politischen Debatten immer größeres Interesse.

Wie sich vor diesem Hintergrund die einschlägige Forschungslandschaft aktuell darstellt, d. h. wer sich aus welchem (disziplinären/institutionellen) Blickwinkel mit welchem Verständnis und zu welchem Zweck der Thematik annimmt, sind einige der Fragen, die diese Schwerpunktausgabe erstmalig für Österreich in den Blick nimmt. Grundlegende Sortierungen dieser Art sind auch deshalb angebracht, weil zwar durchaus relevante Forschung dazu in Österreich auszumachen ist, sich aber noch kein wirklich entwickeltes und vernetztes Forschungsfeld etabliert hat. Die vor diesem Hintergrund wünschenswerte interdisziplinäre Bestandsaufnahme zur Well-Being-Forschung in Österreich kann das Special Issue selbstverständlich nur schlaglichtartig leisten. Dennoch lassen die hier zusammengeführten Beiträge unseres Erachtens sehr gut die beachtliche inhaltliche, konzeptionell-theoretische und methodische Breite erahnen, in der Well-Being-Forschung inzwischen auch in Österreich betrieben wird.

Bereits der erste Beitrag von Leßmann und Buchner, der die Ergebnisse des österreichischen Teilprojekts eines EU-Forschungsvorhabens vorstellt, spiegelt einiges der Interdisziplinarität wider, die das Forschungsfeld auch als Ganzes prägt. Mit Hilfe partizipativer Aktionsforschung und unter Rückgriff auf den Capability- sowie den Menschenrechtsansatz als normativen Rahmen wird den Auswirkungen der Finanz- und Wirtschaftskrise auf das Wohlergehen älterer Arbeitssuchender nachgegangen.

Im Anschluss daran behandeln Hofbauer und Schwingsmehl auf der Grundlage einer breit angelegten, telefonisch durchgeführten quantitativen Befragung im Bundesland Salzburg die Frage, inwiefern hohe Arbeitszufriedenheit - verstanden als ein wichtiger Aspekt des Wohlbefindens Erwerbstätiger - tatsächlich auf gute Arbeitsbedingungen schließen lässt.

Gestützt auf qualitative Tiefeninterviews untersuchen Omann, Mock, Lauer und Schuler den Einfluss bürgerlichen Engagements im Nachhaltigkeitsbereich auf das Wohlbefinden. Dabei werden nicht nur unterschiedliche Nachhaltigkeitsinitiativen berücksichtigt, sondern auch einige der Spezifika reflektiert, denen nachhaltigkeitsbezogenes Engagement unterliegt.

Keul, Brunner und Blaschke widmen sich in ihrem englischsprachigen Beitrag der Messbarkeit der Lebensqualität im urbanen Raum aus umweltpsychologischer Perspektive. Ihr Hauptaugenmerkt liegt dabei auf dem Ineinandergreifen objektiver, auf geoinformatische Daten gestützter, und subjektiver, auf psychologischen Qualityof-Life-Daten gründender Deskriptoren.

Das Finale dieser Sonderausgabe bildet im Anschluss an diese vier ausdrücklich forschungsbasierten Artikel der stärker programmatisch ausgerichtete Beitrag von Georg Feigl. Dabei handelt es sich nicht zuletzt um ein Plädoyer, der Fachdebatte um zeitgemäßere Formen der Wohlstandsmessung künftig in der österreichischen Wirtschaftspolitik stärker Rechnung zu tragen. Feigl liefert darüber hinaus auch einen eigenen Diskussionsvorschlag, wie dies konkret realisiert werden könnte.

Wir hoffen, dass es uns mit dieser Zusammenstellung gelungen ist, in einer breiteren fachinteressierten Öffentlichkeit sowohl Neugier zu wecken als auch ein stärkeres Bewusstsein für die Relevanz und das große gesellschaftspolitische Potenzial der Well-Being-Forschung für Österreich zu befördern. Wenn wir damit auch einen wirksamen Impuls zur weiteren Erschließung und damit vielleicht sogar zur Weiterentwicklung der entsprechenden Forschungslandschaft geleistet hätten, wäre unser Ziel schon erreicht.

Bernhard Babic, Franz Eiffe, Kathrin Gärtner, Ivo Ponocny 